\title{
Joint coding in parallel symmetric interference channels with deterministic model
}

\author{
Yifan Xue, Yafei Tian ${ }^{*}$ and Chenyang Yang
}

\begin{abstract}
In parallel interference channels, the sum-rate achieved by joint coding among subchannels can exceed the sum of the achievable rate of each subchannel with individual coding. In this paper, a capacity-achieving joint coding scheme is proposed for parallel symmetric interference channel. First, we provide a motivating example, from which the insights into the joint coding scheme are obtained. Second, we introduce a transmission scheme in two-user parallel symmetric interference channels, where the subchannels can cooperate to cancel interference. Then, by taking advantage of signal level alignment of the interference from different users, we generalize the scheme to multi-user cases. Finally, we prove that our scheme can achieve the sum capacity and illustrate the generalized degrees of freedom gains over individual coding in various interference scenarios.
\end{abstract}

Keywords: Deterministic model, Generalized degrees of freedom, Interference alignment, Joint coding, Parallel interference channel

\section{Introduction}

Parallel interference channel is a collection of subchannels where each subchannel is an interference channel but there is no interference between the subchannels. The typical parallel interference channels are frequencyselective orthogonal multicarrier interference channel and time-varying multi-symbol interference channel.

When considering parallel interference channel, many researchers focused their attentions on separate coding over each subchannel [1-3]. This might be due to the well-known fact that parallel point-to-point channel, multiple-access channel and broadcast channel are all separable. However, parallel interference channel is not separable in general. As shown by a counterexample in [4], joint coding across multiple subchannels outperforms individually optimal coding. Recently, for the two-user parallel Gaussian interference channels, Shang et al. [5] determined the conditions on the channel coefficients and power constraints under which independent coding across subchannels (i.e., treating interference as noise) is optimal. For $K$-user parallel deterministic interference

*Correspondence: ytian@buaa.edu.cn

School of Electronics and Information Engineering, Beihang University, 37

Xueyuan Road, Haidian District, Beijing 100191, China networks, Sun and Jafar [6] derived the conditions under which treating interference as noise at each subchannel is optimal. For two-user ergodic Gaussian interference channels, Sankar et al. [7] showed that under certain conditions, joint coding across the fading states, which can be seen as subchannels, is required for optimality.

In this paper, the link between a transmitter and its desired receiver is denoted as direct-link, and the link between a transmitter and its undesired receiver is denoted as cross-link. For individual coding in each subchannel, the existence of cross-link can only deteriorate the direct-link transmission [8-10]. In weak interference channels, the interference will decrease the signal-tointerference-plus-noise ratio (SINR). In strong interference channels, although the interference can be decoded and then canceled, it occupies higher signal amplitude levels, leading to a reduced transmission rate of desired signals.

Recently, research results change this pessimistic point of view and reveal that the interference can actually be exploited to help decoding. In [11], the linear interference network problem is translated to the index coding problem. The authors used an example to show that the interference bit decoded in the second time slot can be used to cancel the interference encountered in the first 
time slot. In $[12,13]$, the interference signal goes through two cross-links through feedback and finally becomes desired signal.

In parallel interference channels, the strong interference subchannels can be used to help the decoding of other weak interference subchannels by retransmitting the bits that generate interference, as will be explained in the subsequent sections. However, when two users retransmit their information simultaneously in the same strong interference subchannel, they also interfere with each other. In [14], this problem was simplified by only using individual coding on strong interference subchannels. In [15], considering a specific scenario, only one user was allowed to transmit on these strong interference subchannels. In [16], a capacity-achieving joint coding scheme was proposed for two-user two-subchannel symmetric deterministic interference channel, but the proof of capacity achieving is divided into multiple subcases, and the corresponding joint coding schemes are respectively designed for each subcase. Due to the lack of systematic design principle, this scheme is hard to be generalized to parallel interference channel with multiple subchannels and multiple users. In [17], the parallel symmetric two-user interference channels were studied when the interference is bursty and feedback is available from the receivers. With the help of channel bursty and feedback, the subchannel in very strong interference regime can help to recover the signals for subchannels in strong and weak interference regime. However, the scheme does not work in constant interference channels, and there is no mechanism to let the strong interference subchannel help the weak interference subchannel.

In this paper, we study joint coding problem over parallel symmetric interference channel with multiple subchannels, where each subchannel is constant over the coding block and no feedback information from the receiver is available. First, we introduce a transmission scheme under two-user parallel symmetric deterministic interference channels. Then, we extend the scheme to multi-user cases using the principle of signal level alignment. To prove the optimality of this scheme, we derive the capacity of this class of channels by using El Gamal and Costa's result [18] and show that the proposed scheme can achieve the sum capacity. Finally, we illustrate the generalized degrees of freedom (GDoF) gains of the proposed joint coding scheme over the individual coding scheme in various interference scenarios.

The capacity or GDoF analyses of the interference channel through the help of deterministic channel model have been got a lot of attentions in recent years, but few of them studied the parallel interference channel. For example, in [19], the sum capacity of a special case of $K$-user Gaussian interference network is determined within $O(K)$ bits, where only one of the users interferes with and is also interfered by all the other users. Multi-user cognitive interference network is studied in [20], where secondary users have a priori non-causal message knowledge of primary license holders and can transmit signals to neutralize the interference appeared in primary receivers. Symmetric interference relay channel is studied in [21], where a full-duplex relay is present to coordinate the interference. Furthermore, multicoding scheme is developed in [22], by which the same rate region compared with HanKobayashi coding is achieved in two-user discrete memoryless interference channel. In [23], a tight converse for two-user deterministic interference channel is derived by extended network and generalized cut-set bound.

The rest of this paper is organized as follows. In Section 2, we provide the deterministic model for $K$ user parallel symmetric interference channels. Then, in Section 3, we introduce the individual coding scheme and its achieved GDoF for symmetric interference channels. In Section 4, the joint coding schemes are developed in two-user and multi-user cases, respectively. We prove the optimality of the proposed joint coding scheme in Section 5, where the GDoF gains over individual coding are illustrated through analysis and numerical results. Finally, Section 6 concludes this paper.

\section{Channel model}

It is hard to study the parallel interference network problem under Gaussian channels. In this paper, we resort to the deterministic channel model proposed in [24], which approximates the Gaussian channel as a discrete set of parallel noiseless channels [24, 25].

For the convenience of readers, we first introduce the deterministic model of point-to-point channel proposed in [24] and define several notions to be used subsequently. Then, we introduce the deterministic model of interference channel, in which each link is modeled in the same way as in point-to-point channel. Finally, we provide the deterministic model of parallel symmetric interference channel, in which each subchannel is modeled as in interference channel.

\subsection{Deterministic model of point-to-point channel}

In point-to-point Gaussian channel, a real-valued input $x$ generates a real-valued output $y$ degraded by Gaussian noise $z$, i.e.,

$$
y=h x+z
$$

where $h$ is the channel coefficient, $E\left[|x|^{2}\right]=P$, and the variance of $z$ is $N_{0}$. The signal-to-noise ratio (SNR) is defined as $|h|^{2} P / N_{0}=\gamma$. If the powers of $x$ and $z$ are normalized to 1 , then the effective channel gain is $\sqrt{\gamma}$.

To transform the Gaussian channel to a deterministic channel, we first represent the normalized $x$ in a base- 2 
notation as

$$
\bar{x}=0 . b_{1} b_{2} b_{3} b_{4} b_{5} \ldots,
$$

where each bit $b_{i} \in\{0,1\}$, which can be interpreted as occupying a signal level, and the most significant bit corresponds to the highest signal level.

Definition 1 (signal level) In a deterministic channel, we quantize signal into multiple layers. Each layer is referred to a signal level, or simply called a level.

Definition 2 (bit) Each part of the signal in one level is called a bit. Obviously, all the bits compose the signal.

Definition 3 (relative level order) Different bits from a signal occupy different levels. Relative level order of two bits refers to the relative height between the two levels that they occupy. There are three kinds of relative level order between a pair of bits: higher, lower, and equal.

Given the SNR $\gamma$, the output of the deterministic channel is

$$
\bar{y}=\left\lfloor 2^{M} \bar{x}\right\rfloor=b_{1} b_{2} \ldots b_{M}
$$

where $M=\frac{1}{2}\left\lfloor\log _{2} \gamma\right\rfloor$ is the largest integer below $\log _{2} \gamma$ and $b_{M}$ is the lowest signal level above the noise. In other words, the input bit sequence is shifted by $M$ positions and the remaining part after $b_{M}$ is truncated due to the degradation of noise.

Since there is no interference, the receiver can obtain $M$ bits. The capacity of deterministic point-to-point channel is defined as

$$
C_{\mathrm{P} 2 \mathrm{P}}(\mathrm{SNR})=\frac{1}{2}\left\lfloor\log _{2} \gamma\right\rfloor=M .
$$

The shifting and truncation operations are illustrated in Fig. 1. In Fig. $1, b_{1}, b_{2} \ldots$ are bits occupying different levels. For example, the relative order between $b_{2}$ and $b_{5}$ is that $b_{2}$ is higher than $b_{5}$. Besides, the capacity of this channel is $C_{\mathrm{P} 2 \mathrm{P}}(\mathrm{SNR})=4$.

\subsection{Deterministic model of interference channel}

In $K$-user Gaussian interference channels, the inputs of $K$ users form a vector $\boldsymbol{x}$, and the output vector is

$$
\boldsymbol{y}=\boldsymbol{H} \boldsymbol{x}+\boldsymbol{z},
$$

where the entry of channel matrix $H_{i, j}$ stands for the channel gain from transmitter $j$ to receiver $i$. The noise of different users is assumed to be independent and identically distributed (i.i.d.), and $E\left[z z^{H}\right]=N_{0}$ I. The SNRs depend on the channel gains of the direct-link, which are $\gamma_{k, k}=\left|H_{k, k}\right|^{2} P_{k} / N_{0}$. The interference-to-noise ratios (INRs) depend on the channel gains of the cross-link, which are $\gamma_{i, j}=\left|H_{i, j}\right|^{2} P_{j} / N_{0}, i \neq j$.

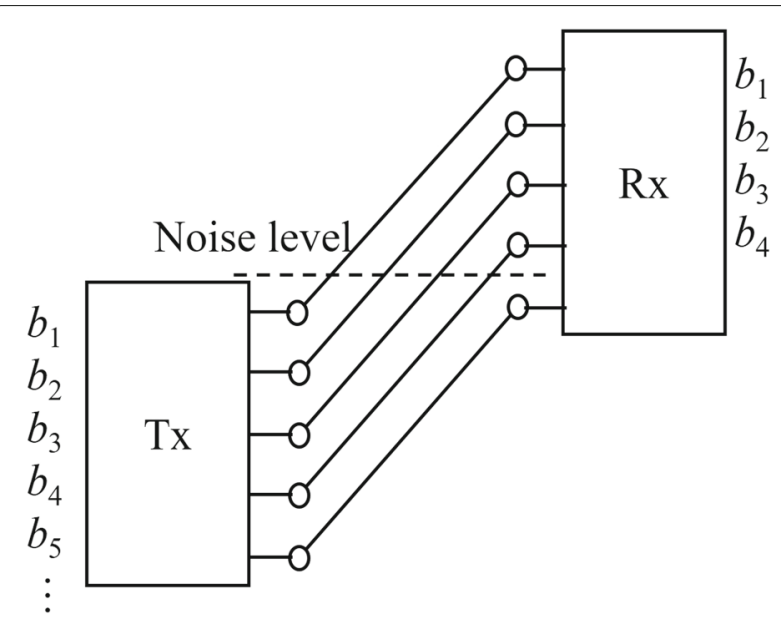

Fig. 1 Deterministic model of a point-to-point channel

For a $K$-user deterministic interference channel, the channel inputs can be written in a base- 2 notation, i.e.,

$$
\begin{aligned}
\bar{x}_{1} & =0 . a_{1} a_{2} a_{3} a_{4} a_{5} \ldots, \\
\bar{x}_{2} & =0 . b_{1} b_{2} b_{3} b_{4} b_{5} \ldots, \\
& \vdots \\
\bar{x}_{K} & =0 . k_{1} k_{2} k_{3} k_{4} k_{5} \ldots,
\end{aligned}
$$

where $a_{i}, b_{i}, \cdots, k_{i} \in\{0,1\}$.

At the receiver, the outputs of the direct-link channel and cross-link channels are added together. Specially, the signal addition takes the form of XOR, i.e., modulo- 2 addition. Therefore, the addition of signal and interference on one signal level does not affect that on other signal levels. The bits that are lower than noise level are lost in this model. This simplification allows us to more focus on the interactions between signal and interference.

Define $M_{i, j}=\frac{1}{2}\left\lfloor\log _{2} \gamma_{i, j}\right\rfloor$ and apply (3) to every direct-link and cross-link of (5). Then, the output can be written as

$$
\begin{aligned}
\bar{y}_{1} & =\left\lfloor 2^{M_{1,1}} \bar{x}_{1}\right\rfloor \oplus\left\lfloor 2^{M_{1,2}} \bar{x}_{2}\right\rfloor \oplus \cdots \oplus\left\lfloor 2^{M_{1, K}} \bar{x}_{K}\right\rfloor, \\
\bar{y}_{2} & =\left\lfloor 2^{M_{2,1}} \bar{x}_{1}\right\rfloor \oplus\left\lfloor 2^{M_{2,2}} \bar{x}_{2}\right\rfloor \oplus \cdots \oplus\left\lfloor 2^{M_{2, K}} \bar{x}_{K}\right\rfloor, \\
& \vdots \\
\bar{y}_{K} & =\left\lfloor 2^{M_{K, 1}} \bar{x}_{1}\right\rfloor \oplus\left\lfloor 2^{M_{K, 2}} \bar{x}_{2}\right\rfloor \oplus \cdots \oplus\left\lfloor 2^{M_{K, K}} \bar{x}_{K}\right\rfloor .
\end{aligned}
$$

An example of the two-user deterministic interference channel is shown in Fig. 2, where we denote the direct-link as solid lines and denote the cross-link as dotted lines.

\subsection{Parallel symmetric interference channel}

In a two-user symmetric interference channel, the SNRs of two direct-link channels are identical and the INRs of two cross-link channels are identical, i.e., $\gamma_{1,1}=\gamma_{2,2}$ and $\gamma_{1,2}=$ $\gamma_{2,1}$. In multi-user symmetric interference channel, all the direct-link gains are the same and all the cross-link gains 


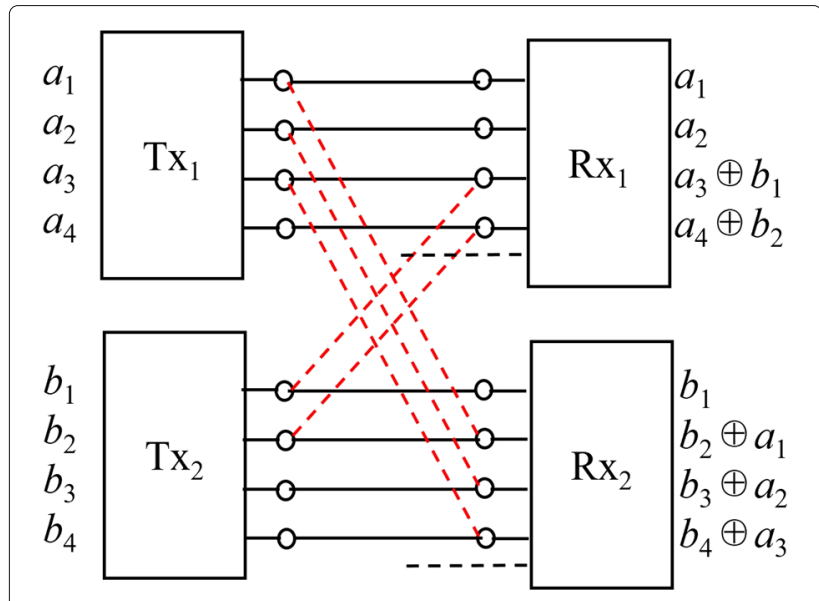

Fig. 2 A two-user deterministic interference channel

are the same as well. In this kind of channel, each user generates interference to other users at the same levels.

In a symmetric interference channel, if the INR is larger than the SNR, we call it a strong interference channel. Otherwise, we call it a weak interference channel. Specifically, in deterministic channels, the strength of interference is expressed by the number of levels. Thus, if there are less cross-link levels than direct-link levels in a deterministic symmetric interference channel, it is a weak interference channel. Otherwise, it is a strong interference channel. For a network with more than one symmetric interference subchannels, we call it a parallel symmetric interference channel, where each subchannel may be a strong or weak interference channel. The difference among subchannels comes from frequency-selective or time-selective fading, i.e., each subchannel may experience different channel fading. An example of a two-user three-subchannel parallel symmetric interference channel is shown in Fig. 5.

In Fig. 5, there is only one transmitter and one receiver for each user. For example, the $\mathrm{Tx}_{1}$ blocks in different subchannels belong to the same transmitter of user 1 .

\section{Individual coding}

Most of previous researches on interference channels focus on individual coding, which means that the coding scheme is taken in each subchannel individually, and there is no cooperation among multiple subchannels. In this section, we will first give an example of the optimal individual coding scheme in a two-user deterministic interference channel and then present the known GDoF results for $K$-user symmetric interference channels.

\subsection{An example of individual coding}

Figure 3 is an example of the individual coding in a two-user deterministic interference channel where the interference conditions are the same as in Fig. 2. It can

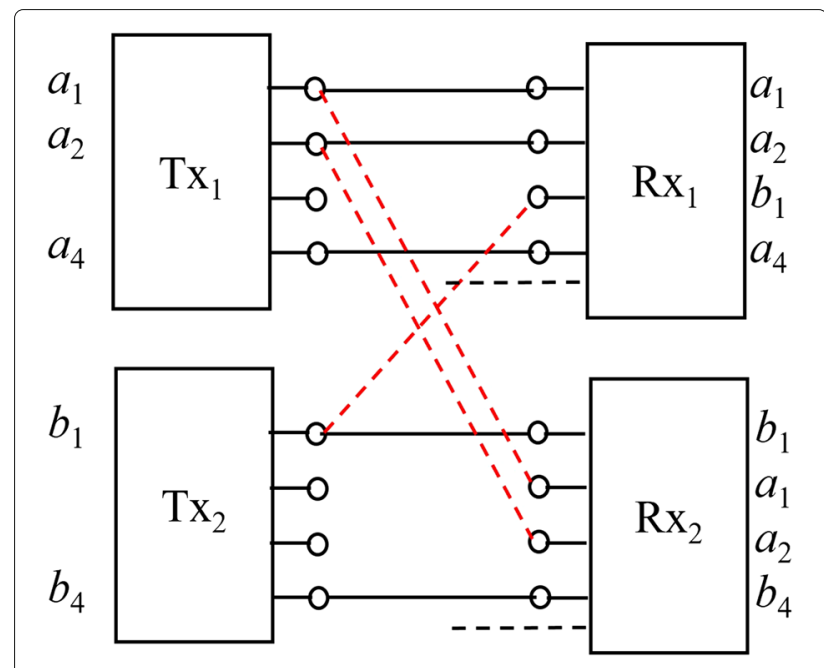

Fig. 3 An example of individual coding

been seen that because of the mutual interference, some signal levels must be muted; otherwise, the superposition bits are not decodable and the system throughput will be degraded. As shown in Fig. 2, if all the signal levels are occupied, only three bits can be decoded for the two users. However, if using the transmission scheme shown in Fig. 3, i.e., user 1 transmits on levels $a_{1}, a_{2}$, and $a_{4}$ and user 2 transmits on levels $b_{1}$ and $b_{4}$, totally five bits can be decoded. Through exhaustive searching, we can find that the sum capacity of this channel is exactly 5 , and the presented scheme in Fig. 3 is thus the best individual coding scheme for this channel.

\subsection{Generalized degrees of freedom}

For the general $K$-user symmetric interference channels, an individual coding scheme is presented in [10]. Although the scheme is originally designed for Gaussian interference channels, the deterministic model is used in the derivations, and it thus can be easily applied in deterministic interference channels. In [10], the GDoF of the $K$-user symmetric Gaussian interference channel is also derived.

The GDoF per user is defined as

$$
d(\alpha)=\frac{1}{K} \lim _{\mathrm{SNR} \rightarrow \infty} \sup \frac{C_{\Sigma}(\mathrm{SNR}, \alpha)}{C_{\mathrm{P} 2 \mathrm{P}}(\mathrm{SNR})},
$$

where $C_{\mathrm{P} 2 \mathrm{P}}(\mathrm{SNR})$ is the interference-free capacity for one user, $C_{\Sigma}(\mathrm{SNR}, \alpha)$ is the sum capacity of the $K$-user interference channel, and

$$
\alpha=\frac{\log \mathrm{INR}}{\log \mathrm{SNR}}
$$

denotes the strength of interference. When $\alpha<1$, it is a weak interference channel; when $\alpha>1$, it is a strong interference channel. 
GDoF denotes the ratio of the average sum capacity of the interference channel normalized by the capacity of the point-to-point interference-free channel when the SNR approaches infinity. For $K$-user symmetric Gaussian interference channels,

$$
C_{\mathrm{P} 2 \mathrm{P}}(\mathrm{SNR})=\frac{1}{2} \log (1+\mathrm{SNR})
$$

and the GDoF is characterized as the following piecewise function [10],

$$
d(\alpha)= \begin{cases}1-\alpha, & \alpha \in\left(0, \frac{1}{2}\right) \text { (noisy weak) } \\ \alpha, & \alpha \in\left(\frac{1}{2}, \frac{2}{3}\right) \text { (fairly weak) } \\ 1-\frac{1}{2} \alpha, & \alpha \in\left(\frac{2}{3}, 1\right) \text { (moderately weak) } \\ \frac{1}{K}, & \alpha=1 \\ \frac{1}{2} \alpha, & \alpha \in(1,2) \text { (moderately strong) } \\ 1, & \alpha \in(2,+\infty) \text { (very strong) }\end{cases}
$$

In this function, the weak interference scenario is further subdivided into three cases and the strong interference scenario is further subdivided into two cases, according to the value of $\alpha$. The piecewise GDoF curve is shown in Fig. 4, where the GDoF achieves maximum in interference-free scenario or very strong interference scenario.

For $K$-user symmetric deterministic interference channels, the GDoF function is as same as in (11), since the difference of the sum capacity in deterministic interference channel and in Gaussian interference channel is within finite bits [10]. When the SNR approaches infinity, the ratio $d(\alpha)$ will go to the same.

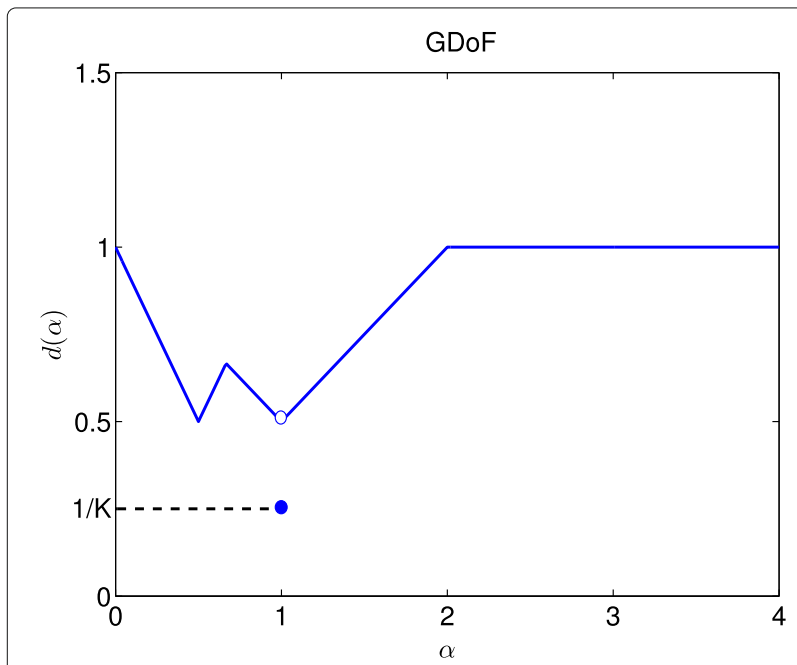

Fig. 4 GDoF of K-user symmetric Gaussian interference channel

\section{Joint coding scheme}

In parallel interference channel, the signals in different subchannels of one user is transmitted by the same transmitter; thus, the subchannels can be jointly encoded. Similarly, the signals received in different subchannels of the same user can be jointly decoded. The basic idea of the proposed transmission scheme is as follows. In weak interference subchannels, the bits is transmitted at the maximal possible data rate of the direct-link without considering the existence of cross-link interference. In strong interference subchannels, the bits that will generate interference in weak interference subchannels is retransmitted following a certain rule. The received signals in weak interference subchannels and strong interference subchannels are then jointly decoded.

\subsection{A motivating example}

To show the basic idea of our joint coding scheme, we first see a simple example. As shown in Fig. 5, the twouser parallel interference channel has three subchannels, we call them subchannels I, II, and III. The number of levels in the direct-link is three in both subchannels I and II. The number of levels in the cross-link is one and two in subchannel I and II, respectively. In subchannel III, two signal levels exist in the direct-link and three signal levels exist in the cross-link. According to the statements in Section 2.3, subchannels I and II are weak interference channels, while subchannel III is a strong interference channel.

In subchannel I, user 1 and user 2 transmit their bits on all signal levels of direct-link regardless of interference. Specifically, $\mathrm{Tx}_{1}$ transmits $a_{1}, a_{2}$, and $a_{3}$ and $\mathrm{Tx}_{2}$ transmits $b_{1}, b_{2}$, and $b_{3}$. Obviously, there are interference at the two receivers as can be seen from Fig. $5 \mathrm{a}$. In particular, since the number of direct-link signal levels $M_{1,1}=3$ and that of cross-link signal levels $M_{2,1}=1$, a bit $a_{1} \oplus b_{3}$ is received at $\mathrm{Rx}_{2}$, which means that $b_{3}$ is interfered by $a_{1}$. Because the channel is symmetric for two users, the interference scenario is similar at $\mathrm{Rx}_{1}$, i.e., $a_{3}$ is interfered by $b_{1}$. Provided the received bits in subchannel I, only $a_{1}$ and $a_{2}$ are decodable at $\mathrm{Rx}_{1}$ and only $b_{1}$ and $b_{2}$ are decodable at $\mathrm{Rx}_{2}$. The contaminated bits $a_{3}$ and $b_{3}$ cannot be decoded without external help.

Subchannel II is also a weak interference channel, we use similar transmission strategy as in subchannel I. The number of cross-link signal levels of this subchannel is larger, $M_{1,2}=M_{2,1}=2$. Therefore, although three bits are still transmitted for each user, two bits are interfered at each receiver, and only $a_{4}$ and $b_{4}$ can be decoded.

Since the bits are transmitted regardless of interference in weak interference subchannels, different bits can be transmitted in subchannel II and subchannel I, and they are independent. This property is essential in our joint coding scheme as we will see in the sequel. 


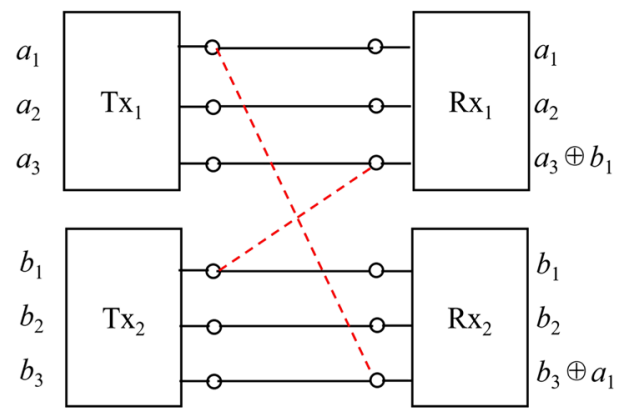

(a)
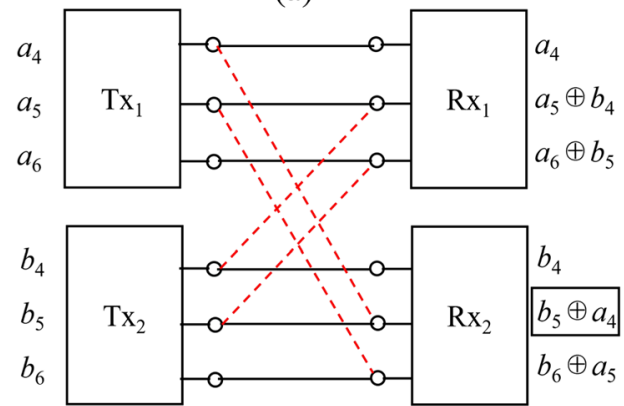

(b)

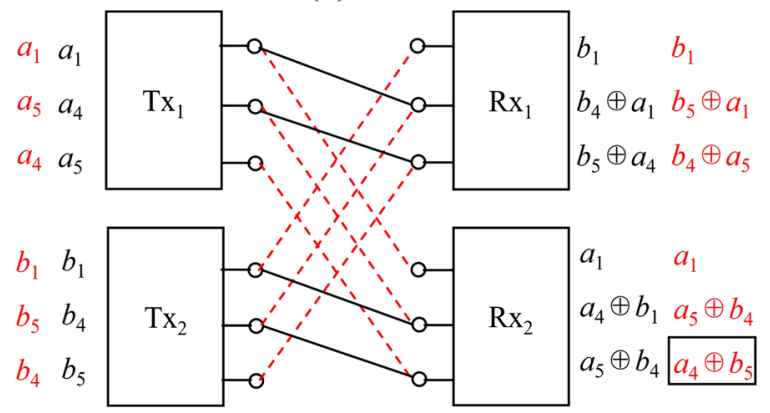

(c)

Fig. 5 A two-user parallel interference channel with three subchannels. a Subchannel I. b Subchannel II. c Subchannel III

Subchannel III is a strong interference channel, where three signal levels exist in the cross-link channel. The transmission scheme in this subchannel is critical. It determines whether the contaminated bits in subchannels I and II are decodable, and affects the spectrum utilization efficiency of the parallel interference channel.

The bits that will generate interference in weak interference subchannels are retransmitted in subchannel III, which is used to recover the contaminated bits in subchannel I and subchannel II. To avoid the interference between user 1 and user 2 in retransmission, a straightforward scheme at hand is orthogonal-based transmission schemes, so that there is no interference between user 1 and user 2. For example, in the first time slot, $\mathrm{Tx}_{1}$ retransmits $a_{1}, a_{4}$, and $a_{5}$. Through the cross-link, these bits arrive at $\mathrm{Rx}_{2}$ and can be used to cancel the interference appeared in subchannels I and II. In the second time slot, $\mathrm{Tx}_{2}$ retransmits $b_{1}, b_{4}$, and $b_{5}$, and $\mathrm{Rx}_{1}$ uses these bits for interference cancelation. However, this scheme is obviously inefficient.

A better choice is to let $\mathrm{Tx}_{1}$ and $\mathrm{Tx}_{2}$ retransmitting simultaneously in subchannel III. As shown in Fig. 5c, both $\mathrm{Rx}_{1}$ and $\mathrm{Rx}_{2}$ obtain contaminated bits and cannot recover these bits individually. However, taking into account the received bits in subchannels I and II, these bits can be jointly decoded. For $\mathrm{Rx}_{1}$, in three subchannels, totally nine bits are obtained, which can be expressed as follows:

$$
\begin{aligned}
& r_{11}=a_{1}, \\
& r_{12}=a_{2}, \\
& r_{13}=a_{3} \oplus b_{1}, \\
& r_{14}=a_{4}, \\
& r_{15}=a_{5} \oplus b_{4}, \\
& r_{16}=a_{6} \oplus b_{5}, \\
& r_{17}=b_{1}, \\
& r_{18}=b_{4} \oplus a_{1}, \\
& r_{19}=b_{5} \oplus a_{4},
\end{aligned}
$$

where $r_{1 i}, i=1, \cdots, 9$ is the received bits at $\mathrm{Rx}_{1}$. We can see from (12) that each received bit correspond to an equation and all the received bits provide us with a set of equations.

It can be seen that $a_{1}, a_{2}, a_{4}$, and $b_{1}$ can be obtained immediately when $r_{11}, r_{12}, r_{14}$, and $r_{17}$ is received. But the other five received bits are not simply transmitted bits from user 1 or user 2, none of which can be recovered by a single equation. Fortunately, the nine equations in (12) are linear uncorrelated, and there are only nine unknown variables in (12). By solving the set of equations, all the nine bits can be recovered. In these bits, six are transmitted by user 1 , which are the desired bits. The other three bits, which are transmitted by user 2 to facilitate interference cancelation, will be discarded after decoding. Since the channel is symmetric, similar characteristic holds for $\mathrm{Rx}_{2}$.

Remark 1 In subchannel III, the order of the retransmission cannot be arbitrary. For example, if we exchange the occupied levels of $a_{4}$ and $a_{5}$, as labeled in the outer column in Fig. $5 c, a_{4} \oplus b_{5}$ will be received twice at $R x_{2}$. One is obtained in subchannel II, and the other is obtained in subchannel III, as indicated by the black box. In this case, part of the equations are linearly correlated, and the desired bits cannot be fully decoded.

\subsection{Subchannel grouping}

In the example above, the cross-link signal levels of weak interference subchannels are as many as that of strong interference subchannel. This condition is obviously not satisfied in most scenarios. Under the condition where there are more cross-link signal levels in weak interference 
subchannels than that of strong interference subchannels, the resource to recover the bits contaminated in weak subchannels is not enough. Under opposite condition, the resource is too much and will be wasted. Therefore, a preprocessing step called subchannel grouping is introduced.

Denote the total number of cross-link signal levels of all the weak interference subchannels as $N_{\text {weak }}$ and that of all the strong interference subchannels as $N_{\text {strong. If }} N_{\text {weak }}>$

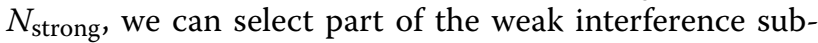
channels to participate joint coding. The aggregated number of cross-link signal levels of this part of subchannels is

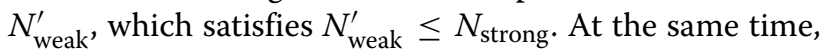
other weak interference subchannels employ individual coding introduced in Section 3. If $N_{\text {weak }}<N_{\text {strong, we }}$ can select part of the strong interference subchannels to participate joint coding. The aggregated number of crosslink signal levels of this part of subchannels is $N_{\text {strong, }}^{\prime}$, which still satisfies $N_{\text {strong }}^{\prime} \geq N_{\text {weak. }}$. At the same time, other strong interference subchannels employ individual coding. As will be seen in next part, subchannel grouping ensures to satisfy a necessary condition under which the bits in the subchannels participating joint coding can be jointly decoded.

\subsection{Joint coding scheme for two-user case}

After the subchannels for joint coding are selected, in weak interference subchannels, all the direct-link signal levels are used to transmit new bits regardless of interference. In strong interference subchannels, the bits that will generate interference in weak interference subchannels, i.e., interfering bits, are retransmitted. It is demanded that in the retransmission process, the relative level orders between any pair of bits are kept unchanged compared with the orders when they are transmitted in the weak interference subchannels.

The joint coding scheme ensures the feasibility that the transmitted bits can be jointly decoded. In the following, we will formally prove the necessary condition and the feasibility of the proposed transmission scheme. As we will see, the necessary condition guarantees that there are enough number of equations, while the feasibility comes from the requirement that these equations are linearly uncorrelated. When enough number of linearly uncorrelated equations are obtained, the bits can be decoded.

Necessary Condition For subchannels participating joint coding, the aggregated cross-link signal levels of strong interference subchannels should be no less than the aggregated cross-link signal levels of weak interference subchannels.

Proof To ensure the interference in weak interference subchannels which can be eliminated with the help of strong interference subchannels, at each receiver, the number of equations should be no less than the number of desired bits and interfering bits. Without loss of generality, we consider user 1 . Assume that in all weak interference subchannels, there are totally $X$ signal levels in the directlink and $Y$ signal levels in cross-links. Then, we have $X+Y$ unknown bits but only have $X$ linear equations. To decode these bits, we need at least $Y$ more linear uncorrelated equations, which should be provided by the crosslink retransmission in strong interference subchannels. Thus, the number of the aggregated signal levels of crosslinks in strong interference subchannels should be no less than $Y$.

Feasibility By the proposed joint coding scheme, the desired bits can be recovered at each receiver.

Proof The cornerstone of this proof is the fact that the transmit levels of interfering bits are always lower than those of the desired bits in weak interference subchannels and vice versa in strong interference subchannels at receivers.

Assume that in a weak interference subchannel $S_{1}, \mathrm{Tx}_{1}$ transmits bit $a_{m}$ and $\mathrm{Tx}_{2}$ transmits bit $b_{n}$, these two bits collide on the same signal level at $\mathrm{Rx}_{2}$. The bit $a_{m}$ will be retransmitted in strong interference subchannel $S_{2}$ and might collide with a bit $b_{p}$ at $\mathrm{Rx}_{2}$. The bit $b_{p}$ is first transmitted by $\mathrm{Tx}_{2}$ in a weak interference subchannel $S_{3}$ and is retransmitted by $\mathrm{Tx}_{2}$ in $S_{2}$. There are two possible cases when we check the linear correlation property between $a_{m} \oplus b_{n}=r_{2 k}$ and $a_{m} \oplus b_{p}=r_{2 j}$, where $m, n, p, k, j$ are integers.

The first case is that $S_{3} \neq S_{1}$, i.e., $b_{p}$ and $b_{n}$ come from different weak interference subchannels. This suggests that they are different bits, for independent bits are transmitted in different weak interference subchannels. Hence, the two equations $a_{m} \oplus b_{n}=r_{2 k}$ and $a_{m} \oplus b_{p}=r_{2 j}$ are linearly uncorrelated.

The second case is that $S_{3}=S_{1}$, i.e., $b_{p}$ and $b_{n}$ come from the same weak interference subchannel. In what follows, we show that they must occupy different signal levels at $\mathrm{Tx}_{2}$.

Assume that $a_{m}$ is transmitted on the $m$ th level of $\mathrm{Tx}_{1}$ in $S_{1}$ and retransmitted on the $m^{\prime}$ th level of $\mathrm{Tx}_{1}$ in $S_{2} . b_{n}$ is transmitted on the $n$th level of $\mathrm{Tx}_{2}$ in $S_{1}$ and retransmitted on the $n^{\prime}$ th level of $\mathrm{Tx}_{2}$ in $S_{2} . b_{p}$ is transmitted on the $p$ th level of $\mathrm{Tx}_{2}$ in $S_{1}$ and retransmitted on the $p^{\prime}$ th level of $\mathrm{Tx}_{2}$ in $S_{2}$. In weak interference subchannels, SNR > INR; thus, we have $m<n$. In strong interference subchannels, SNR < INR; thus, we have $m^{\prime}>p^{\prime}$. Since the relative order in the retransmission process is the same with that in the first transmission, from $m^{\prime}>p^{\prime}$, we can derive $m>p$. Finally, we have the relationship $n>m>p$, which means $b_{p}$ and $b_{n}$ comes from different 
signal level at $\mathrm{Tx}_{2}$, and they are two different independent bits. As a consequence, the two equations $a_{m} \oplus b_{n}=r_{2 k}$ and $a_{m} \oplus b_{p}=r_{2 j}$ must be linear uncorrelated, and all the bits can be recovered.

To help understand the proof, we provide an example here. As shown in Fig. 6, $S_{1}$ and $S_{2}$ are respectively a weak interference subchannel and a strong interference subchannel, and $S_{3}=S_{1}$. In this example, suppose that $a_{m}=a_{2}$, then it follows that $b_{n}=b_{3}$ and $b_{p}=b_{1}$. In subchannel $S_{1}$, since SNR > INR, $a_{2}$ and $b_{3}$ collide on the same signal level at $\mathrm{Rx}_{2}$. In subchannel $S_{2}$, since SNR $<$ INR, $a_{2}$ and $b_{1}$ collide on the same signal level at $\mathrm{Rx}_{2}$. It follows that $a_{2} \oplus b_{3}$ and $a_{2} \oplus b_{1}$ are uncorrelated. The bits $a_{k}$ and $b_{j}$ in Fig. 6(b) are first transmitted in other weak interference sunchannels, which are not shown here.

\subsection{Joint coding scheme for multi-user case}

In a multi-user symmetric interference channel, all the direct-link channel gains are identical and so are the crosslink gains. As shown in Fig. 7, for a $K$-user symmetric interference channel, each user receives interference from other $K-1$ users. Due to the symmetry, at each receiver,

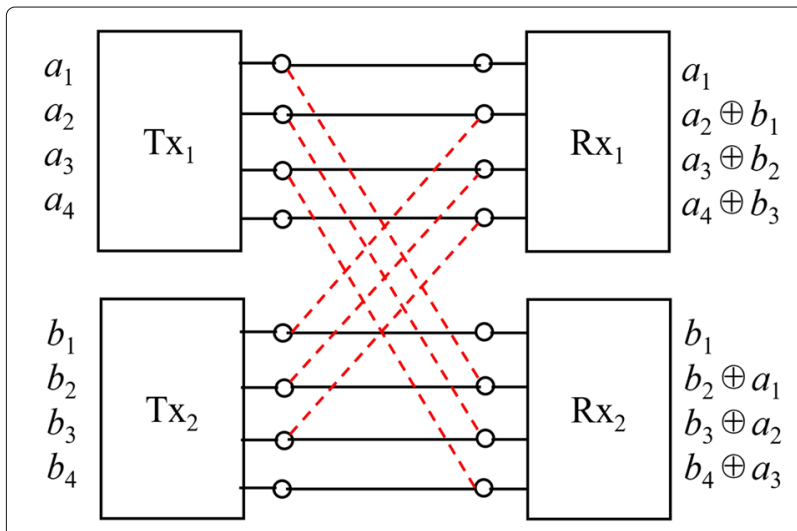

(a)

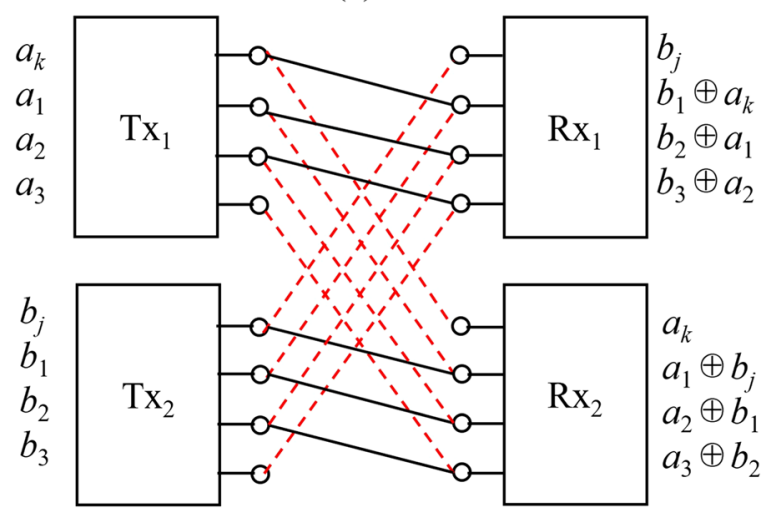

(b)

Fig. 6 An example to illustrate the feasibility. a Subchannel $S_{1}$. b Subchannel $S_{2}$

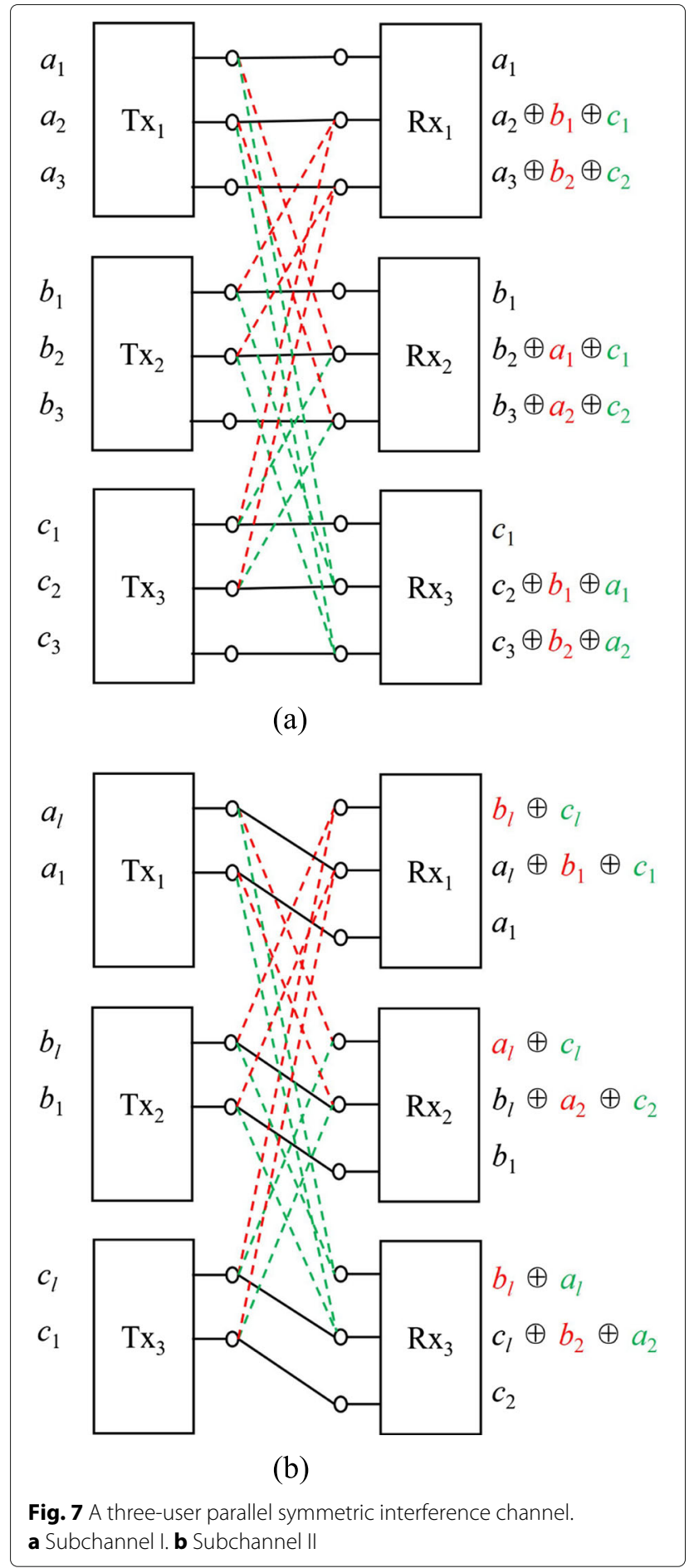

the interference signal levels are aligned. If we view the aligned interference as coming from a virtual user, the joint coding and decoding in the multi-user case can be implemented as same as in the two-user case.

The proofs of necessary condition and feasibility for multi-user joint coding are the same as in the two-user case, except that we view the aligned interference from 
$K-1$ users as coming from one virtual user. Figure 7 is an example of a three-user parallel symmetric interference channel, where only two subchannels are shown. Subchannel I is a weak interference subchannel, and subchannel II is a strong interference subchannel. In subchannel $\mathrm{I}$, as can be seen in Fig. 7a, the modulo- 2 addition of $a_{2} \oplus b_{1} \oplus c_{1}=r_{12}$ is received on the second level of $\mathrm{Rx}_{1}$, where $a_{2}$ is the desired bit and $b_{1} \oplus c_{1}$ is the interference bit. We can regard $b_{1} \oplus c_{1}$ as one bit $u_{1}$ that is transmitted by a virtual user $\mathrm{Tx}_{u}$. Then, we obtain an equation $a_{2} \oplus u_{1}=r_{12}$. In subchannel II, as can be seen in Fig. 7b, $b_{1}$ and $c_{1}$ are retransmitted by $\mathrm{Tx}_{2}$ and $\mathrm{Tx}_{3}$, respectively, and the virtual interference bit $u_{1}=b_{1} \oplus c_{1}$ will reappear at $\mathrm{Rx}_{1}$ with a desired bit $a_{l}$, where $a_{l}$ is a retransmission bit that causes interference in another weak interference subchannel which is not shown here. Then, we obtain another equation $a_{l} \oplus u_{1}=r_{12}^{\prime}$. Since the levels of interfering bits are always lower than the levels of desired bits in weak interference subchannels and vice versa for strong interference subchannels at receivers, even though $a_{2}$ is also retransmitted in this strong interference subchannel, the relative level order of $a_{l}$ and $a_{2}$ cannot be equal at $\mathrm{Tx}_{1}$. Thus, the two equations $a_{2} \oplus u_{1}=r_{12}$ and $a_{l} \oplus u_{1}=r_{12}^{\prime}$ are linear uncorrelated. Then, the desired bits of user 1 can be jointly decoded at $\mathrm{Rx}_{1}$. Similar equations can be obtained at $\mathrm{Rx}_{2}$ and $\mathrm{Rx}_{3}$, and in this way, we generalize the joint coding scheme to multi-user parallel symmetric interference channels.

\section{Performance analysis}

In the proposed joint coding scheme, the resource of strong interference subchannels are totally sacrificed to help the weak interference subchannels to achieve interference-free transmission, since no new bits are transmitted in strong interference subchannels. Then, a natural question is that under what conditions will this scheme have performance gain over the individual coding? Is this scheme optimal? We answer these questions in this section.

\subsection{Sum capacity}

Theorem 1 For two-user parallel symmetric interference channel with deterministic model, when the number of cross-link levels in weak interference subchannels equals to that in strong interference subchannels, the joint coding scheme will achieve the sum capacity

$$
C_{\Sigma}=\sum_{s \in \mathbb{S}_{\text {weak }}} 2 n_{s}
$$

where $\mathbb{S}_{\text {weak }}$ represents the set of weak interference subchannels, $s$ denotes a weak interference subchannel in $\mathbb{S}_{\text {weak }}$, and $n_{s}$ denotes the number of direct-link signal levels in subchannel s.
Proof We first prove the converse by deriving the sumrate constraints and then prove the achievability by providing the achieved sum rate of the joint coding scheme.

Converse The deterministic model of two-user parallel symmetric interference channel belongs to a class of deterministic interference channel studied by El Gamal and Costa [18]. The El Gamal-Costa model is redrawn in Fig. 8, where the outputs $Y_{1}$ and $Y_{2}$ and the interferences $V_{1}$ and $V_{2}$ are deterministic functions of the inputs $X_{1}$ and $X_{2}$ :

$$
\begin{aligned}
& Y_{1}=f_{1}\left(X_{1}, V_{2}\right), \\
& Y_{2}=f_{2}\left(X_{2}, V_{1}\right), \\
& V_{1}=g_{1}\left(X_{1}\right), \\
& V_{2}=g_{2}\left(X_{2}\right),
\end{aligned}
$$

where $f_{1}(\cdot, \cdot)$ and $f_{2}(\cdot, \cdot)$ satisfy the conditions

$$
\begin{aligned}
& H\left(Y_{1} \mid X_{1}\right)=H\left(V_{2}\right), \\
& H\left(Y_{2} \mid X_{2}\right)=H\left(V_{1}\right),
\end{aligned}
$$

for all product probability distributions on $X_{1} X_{2}$.

In the considered parallel symmetric interference channel, $X_{1}$ represents the transmit bits of all subchannels of user $1, g_{1}\left(X_{1}\right)$ represents the cross-link shifting function over $X_{1}$, and $f_{1}\left(X_{1}, V_{2}\right)$ represents the function that involves direct-link shifting over $X_{1}$ and modulo-2 sum with $V_{2}$. Similar representations are applied to $X_{2}, g_{2}\left(X_{2}\right)$, and $f_{2}\left(X_{2}, V_{1}\right)$. In (15), the conditional entropy of $Y_{1}$ over $X_{1}$ equals to the entropy of $V_{2}$, which means that $X_{1}$ can be uniquely identified from $Y_{1}$ given a determined $V_{2}$. Similarly, $X_{2}$ can be uniquely identified from $Y_{2}$ given a determined $V_{1}$.

The capacity region of this class of deterministic interference channel is characterized as [18]

$$
\begin{aligned}
R_{1} & \leq H\left(Y_{1} \mid V_{2}\right), \\
R_{2} & \leq H\left(Y_{2} \mid V_{1}\right), \\
R_{1}+R_{2} & \leq H\left(Y_{1} \mid V_{1} V_{2}\right)+H\left(Y_{2}\right), \\
R_{1}+R_{2} & \leq H\left(Y_{2} \mid V_{1} V_{2}\right)+H\left(Y_{1}\right), \\
R_{1}+R_{2} & \leq H\left(Y_{1} \mid V_{1}\right)+H\left(Y_{2} \mid V_{2}\right), \\
2 R_{1}+R_{2} & \leq H\left(Y_{1}\right)+H\left(Y_{1} \mid V_{1} V_{2}\right)+H\left(Y_{2} \mid V_{2}\right), \\
2 R_{2}+R_{1} & \leq H\left(Y_{2}\right)+H\left(Y_{2} \mid V_{1} V_{2}\right)+H\left(Y_{1} \mid V_{1}\right) .
\end{aligned}
$$

Considering that there are totally $S$ subchannels, due to the symmetric property of each subchannel, we denote the number of direct-link signal levels of the $s$ th subchannel as $n_{s}$ and the number of cross-link signal levels as $m_{s}$. Following the derivation in [16], the entropies in (16) can be further simplified as

$$
\begin{aligned}
& H\left(Y_{1} \mid V_{2}\right) \leq \sum_{s=1}^{S} H\left(Y_{1, s} \mid V_{2, s}\right) \leq \sum_{s=1}^{S} n_{s}, \\
& H\left(Y_{1}\right) \leq \sum_{s=1}^{S} H\left(Y_{1, s}\right) \leq \sum_{s=1}^{S} \max \left(m_{s}, n_{s}\right),
\end{aligned}
$$




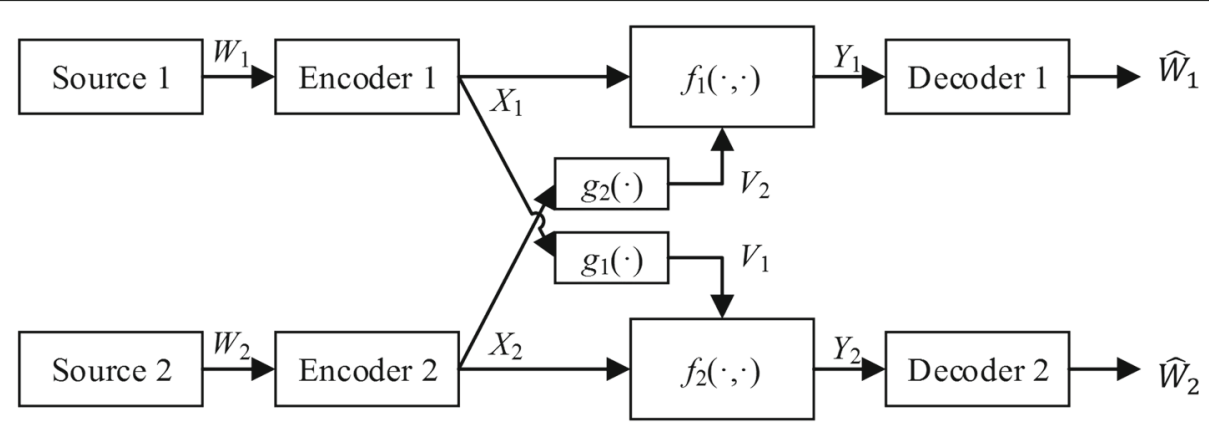

Fig. 8 El Gamal and Costa's deterministic interference channel model

$$
\begin{aligned}
& H\left(Y_{1} \mid V_{1} V_{2}\right) \leq \sum_{s=1}^{S} H\left(Y_{1, s} \mid V_{1, s} V_{2, s}\right) \leq \sum_{s=1}^{S} \max \left(n_{s}-m_{s}, 0\right), \\
& H\left(Y_{1} \mid V_{1}\right) \leq \sum_{s=1}^{S} H\left(Y_{1, s} \mid V_{1, s}\right) \leq \sum_{s=1}^{S} \max \left(n_{s}-m_{s}, m_{s}\right) .
\end{aligned}
$$

Similar results hold for $Y_{2}$. Substituting these results in (16), we can obtain the sum-rate constraints as

$$
\begin{aligned}
R_{1}+R_{2} & \leq H\left(Y_{1} \mid V_{1} V_{2}\right)+H\left(Y_{2}\right) \\
& \leq \sum_{s=1}^{S} \max \left(n_{s}-m_{s}, 0\right)+\sum_{s=1}^{S} \max \left(m_{s}, n_{s}\right) \\
& =\sum_{s=1}^{S} \max \left(m_{s}, 2 n_{s}-m_{s}\right),
\end{aligned}
$$

and

$$
\begin{aligned}
R_{1}+R_{2} & \leq H\left(Y_{1} \mid V_{1}\right)+H\left(Y_{2} \mid V_{2}\right) \\
& \leq \sum_{s=1}^{S} 2 \max \left(n_{s}-m_{s}, m_{s}\right)
\end{aligned}
$$

Considering that in weak interference subchannels, $n_{s}>$ $m_{s}$ and, in strong interference subchannels, $n_{s}<m_{s}$, (21) can be further expressed as

$$
R_{1}+R_{2} \leq \sum_{s \in \mathbb{S}_{\text {weak }}}\left(2 n_{s}-m_{s}\right)+\sum_{s \in \mathbb{S}_{\text {strong }}} m_{s}
$$

where $\mathbb{S}_{\text {weak }}$ and $\mathbb{S}_{\text {strong }}$ represent the set of weak and strong interference subchannels, respectively.

Similarly, (22) can be further expressed as

$$
R_{1}+R_{2} \leq \sum_{s \in \mathbb{S}_{\text {noisy }}} 2\left(n_{s}-m_{s}\right)+\sum_{s \in \mathbb{S}_{\text {medium }}} 2 m_{s}+\sum_{s \in \mathbb{S}_{\text {strong }}} 2 m_{s},
$$

where $\mathbb{S}_{\text {noisy }}$ represents the set of weak interference subchannels satisfying $m_{s} / n_{s}<1 / 2, \mathbb{S}_{\text {medium }}$ represents the set of weak interference subchannels satisfying $1 / 2 \leq$ $m_{s} / n_{s}<1$, and $\mathbb{S}_{\text {strong }}$ represents the set of strong interference subchannels satisfying $m_{s} / n_{s} \geq 1$.

Since the number of cross-link signal levels in weak interference subchannels equals to that in strong interference subchannels, i.e.,

$$
\sum_{s \in \mathbb{S}_{\text {weak }}} m_{s}=\sum_{s \in \mathbb{S}_{\text {strong }}} m_{s}
$$

then (23) can be simplified as

$$
R_{1}+R_{2} \leq \sum_{s \in \mathbb{S}_{\text {weak }}} 2 n_{s}
$$

and (24) can be simplified as

$$
\begin{aligned}
R_{1}+R_{2} \leq & \sum_{s \in \mathbb{S}_{\text {noisy }}} 2\left(n_{s}-m_{s}\right)+\sum_{s \in \mathbb{S}_{\text {medium }}} 2\left(n_{s}-m_{s}\right) \\
& +\sum_{s \in \mathbb{S}_{\text {medium }}}\left(4 m_{s}-2 n_{s}\right)+\sum_{s \in \mathbb{S}_{\text {strong }}} 2 m_{s} \\
= & \sum_{s \in \mathbb{S}_{\text {weak }}} 2 n_{s}+\sum_{s \in \mathbb{S}_{\text {medium }}}\left(4 m_{s}-2 n_{s}\right) .
\end{aligned}
$$

According to the relationship of $m_{s}$ and $n_{s}$ in medium weak interference subchannels, the second term in the last step of (27) is no less than zero, indicating that the constraint (26) is stricter than (27). Thus, the active sum-rate constraint is (26).

Achievability For the proposed joint coding scheme, in weak interference subchannels, users transmit new bits regardless of interference and, in strong interference subchannels, only interfered bits are transmitted. Since the number of cross-link signal levels in weak interference subchannels equals to that in strong interference subchannels, the received bits can be jointly decoded. The achieved sum rate of two users is

$$
R_{1}+R_{2}=\sum_{s \in \mathbb{S}_{\text {weak }}} 2 n_{s} .
$$


Comparing (28) with (26), we conclude that the joint coding scheme achieves the sum capacity.

Theorem 2 For K-user parallel symmetric interference channel with deterministic model, when the number of cross-link levels in weak interference subchannels equals to that in strong interference subchannels, the joint coding scheme achieves a sum rate

$$
R_{\Sigma}=\sum_{s \in \mathbb{S}_{\text {weak }}} K n_{s}
$$

The proof of Theorem 2 is the same as proving the achievability part in Theorem 1 .

While the achievable sum rate of $K$-user parallel symmetric interference channel with deterministic model has been obtained, we do not know the sum capacity of this channel yet. However, we conjecture that the proposed joint coding scheme achieves the sum capacity, since each user achieves a data rate as high as in the two-user interference channels.

\subsection{Achievable GDoF}

To obtain an explicit expression of the achievable GDoF and compare it with the individual coding scheme, we consider a special case of the parallel symmetric interference channel. Assume that the number of direct-link signal levels are $n$ for all the subchannels; the number of cross-link signal levels in weak interference subchannels are $m_{1}$, and those in strong interference subchannels are $m_{2}$. The interference strength of the two kinds of subchannels can be expressed as

$$
\alpha_{1}=\frac{m_{1}}{n}<1, \quad \alpha_{2}=\frac{m_{2}}{n}>1 .
$$

For the proposed joint coding scheme, $\alpha_{1}$ strong interference subchannels can assist $\alpha_{2}$ weak interference subchannels. For convenience of demonstration, the number of weak interference subchannels is normalized to 1 . In this sense, we say one strong interference subchannel can assist to recover the contaminated bits in $\alpha_{2} / \alpha_{1}$ weak interference subchannels. For two-user case, according to Theorem 1, the sum capacity of these $\left(\alpha_{2} / \alpha_{1}+1\right)$ subchannels is

$$
C_{\Sigma}=\sum_{s \in \mathbb{S}_{\text {weak }}} 2 n=2 n \frac{\alpha_{2}}{\alpha_{1}} .
$$

Then, with (8), the GDoF per user is

$$
d_{\text {Joint }}\left(\alpha_{1}, \alpha_{2}\right)=\frac{\alpha_{2}}{\alpha_{1}} \text {. }
$$

For multi-user case, since the achieved sum rate is (29), the joint coding scheme achieves the per user GDoF as same as (31). That means, in $K$-user parallel symmetric interference channels, each user achieves a GDoF which is the same as that can be achieved in two-user interference channels. Thus, for multi-user case, the joint coding scheme is at least GDoF optimal.

\subsection{GDoF gains}

We continually consider the scenario where one strong interference subchannel is used to assist $\alpha_{2} / \alpha_{1}$ weak interference subchannels. By individual coding, the GDoF of the strong interference subchannel is $d\left(\alpha_{2}\right)$ and the GDoF of the weak interference subchannel is $d\left(\alpha_{1}\right)$. Then, the total GDoF of these $\left(\alpha_{2} / \alpha_{1}+1\right)$ subchannels per user is

$$
d_{\text {Indiv }}\left(\alpha_{1}, \alpha_{2}\right)=\frac{\alpha_{2}}{\alpha_{1}} d\left(\alpha_{1}\right)+d\left(\alpha_{2}\right),
$$

where $d\left(\alpha_{1}\right)$ and $d\left(\alpha_{2}\right)$ can be obtained from (11).

Compared with the achieved GDoF by joint coding, the average GDoF gain per subchannel in each user is

$$
\begin{aligned}
\Delta \bar{d}\left(\alpha_{1}, \alpha_{2}\right) & =\frac{d_{\text {Joint }}\left(\alpha_{1}, \alpha_{2}\right)-d_{\text {Indiv }}\left(\alpha_{1}, \alpha_{2}\right)}{\alpha_{2} / \alpha_{1}+1} \\
& =\frac{\frac{\alpha_{2}}{\alpha_{1}}\left[1-d\left(\alpha_{1}\right)\right]-d\left(\alpha_{2}\right)}{\frac{\alpha_{2}}{\alpha_{1}}+1} .
\end{aligned}
$$

In Table 1 , we list the values of $\Delta \bar{d}\left(\alpha_{1}, \alpha_{2}\right)$ under various combinations of $\alpha_{1}$ and $\alpha_{2}$. From the results, we can see that we are able to provide positive GDoF gain when we use very strong interference subchannels to assist all kinds of weak interference subchannels and use moderately strong interference subchannels to assist noisy weak interference subchannels and fairly weak interference subchannels. However, when we use moderately strong interference subchannels to assist moderately weak interference subchannels, no gain can be obtained. Besides, when using very strong interference subchannels to assist moderately strong interference subchannels, we can still obtain positive gain under certain conditions, although this is not the typical scenario that we have studied.

\subsection{Numerical results}

To demonstrate the GDoF gain, we provide some numerical results in this part. We first calculate the average achievable GDoFs when one strong interference subchannel coexists with $\alpha_{2} / \alpha_{1}$ weak interference subchannels. In this example, we fix $\alpha_{2}=3$ and change $\alpha_{1}$ from 0 to 1.5 . Of course, when $\alpha_{1}>1$, the channel no longer belongs to weak interference subchannel. But by setting the parameter in this range, we can obtain more useful insights. Figure 9 shows the results, where the "W" form solid line in blue represents the performance of individual coding and the red dash line represents the performance of the proposed joint coding. Note the blue solid line in Fig. 9 is not so straight as the one in Fig. 4. This comes from the fact that the result in Fig. 9 is obtained by averaging among $\alpha_{2} / \alpha_{1}+1$ subchannels. It can be seen in Fig. 9 when $\alpha_{1} \in$ $(0,1)$, there are positive gains and, when $\alpha_{1}>1$, the gain is 
Table 1 Average GDoF gain per subchannel

\begin{tabular}{lll}
\hline$\Delta \bar{d}\left(\alpha_{1}, \alpha_{2}\right)$ & $\alpha_{2} \in(1,2)$ & $\alpha_{2} \in(2,+\infty)$ \\
\hline$\alpha_{1} \in\left(0, \frac{1}{2}\right)$ & $\frac{\alpha_{1} \alpha_{2}}{2\left(\alpha_{1}+\alpha_{2}\right)} \in\left(0, \frac{1}{5}\right)$ & $\frac{\left(\alpha_{2}-1\right) \alpha_{1}}{\alpha_{2}+\alpha_{1}} \in\left(0, \frac{1}{2}\right)$ \\
$\alpha_{1} \in\left(\frac{1}{2}, \frac{2}{3}\right)$ & $\frac{2 \alpha_{2}-3 \alpha_{1} \alpha_{2}}{2 \alpha_{1}+2 \alpha_{2}} \in\left(0, \frac{1}{5}\right)$ & $\frac{\alpha_{2}-\alpha_{2} \alpha_{1}-\alpha_{1}}{\alpha_{2}+\alpha_{1}} \in\left(0, \frac{1}{2}\right)$ \\
$\alpha_{1} \in\left(\frac{2}{3}, 1\right)$ & 0 & $\frac{\alpha_{1}\left(\alpha_{2}-2\right)}{2\left(\alpha_{2}+\alpha_{1}\right)} \in\left(0, \frac{1}{2}\right)$ \\
$\alpha_{1} \in(1,2)$ & - & $\frac{\alpha_{1}\left(2 \alpha_{2}-\alpha_{1} \alpha_{2}-2 \alpha_{1}\right)}{2\left(\alpha_{2}+\alpha_{1}\right)} \in\left(-\frac{4}{3}, \frac{1}{2}\right)^{*}$ \\
\hline
\end{tabular}

$* \Delta \bar{d}>0$ when $\alpha_{2}>\frac{2 \alpha_{1}}{2-\alpha_{1}}$

still positive within a certain range. Only when $\alpha_{1}$ is larger, the gains become negative. This result indicates that, even when all the subchannels experience strong interference, it still has chance to improve the average achievable GDoF if we use very strong interference subchannels to assist strong interference subchannels.

Now, we provide a more comprehensive result in Fig. 10, where $\alpha_{1}$ varies from 0 to 1 and $\alpha_{2}$ varies from 1 to 4. In this figure, only GDoF gain is drawn, from which the dependency of the gain $\Delta \bar{d}$ over different $\alpha_{1}$ and $\alpha_{2}$ can be seen more clearly. For a fixed $\alpha_{1}, \Delta \bar{d}$ grows monotonically with $\alpha_{2}$. This result comes from the fact that the more cross-link signal levels can be used to employ retransmission in the strong interference subchannel, the more weak interference subchannels can be assisted. For a fixed $\alpha_{2}, \Delta \bar{d}$ varies like an $N$-curve, first increases when $0<\alpha_{1}<\frac{1}{2}$, then decreases when $\frac{1}{2}<\alpha_{1}<\frac{2}{3}$, and finally increases again when $\frac{2}{3}<$ $\alpha_{1}<1$. This is mainly because of the behavior of $d\left(\alpha_{1}\right)$ achieved by the individual coding, as can be seen in Fig. 4. When $0<\alpha_{1}<1, d\left(\alpha_{1}\right)$ is a reverse $N$-curve.
In the cases when the individual coding can achieve high GDoF, the gain of joint coding is relatively low.

In [17], only the subchannels in very strong interference can help to recover the signals for subchannels in strong and weak interference. While in this paper, from the above analysis, we know that the proposed joint coding scheme can also let the strong interference subchannel help the weak interference subchannel. Moreover, only two-user case is considered in [17], but $K$-user case is also considered in this paper. In [17], the interference channel is assumed to be bursty, and the helping mechanism cannot work when the channel is constant. However, the scheme proposed in this paper always works no matter the channel is constant or bursty. In particular, when the channel is bursty, different time slots can be regarded as different subchannels.

\section{Conclusions}

In this paper, a general joint coding scheme in parallel symmetric interference channel with deterministic model was proposed where the cross-links of the strong

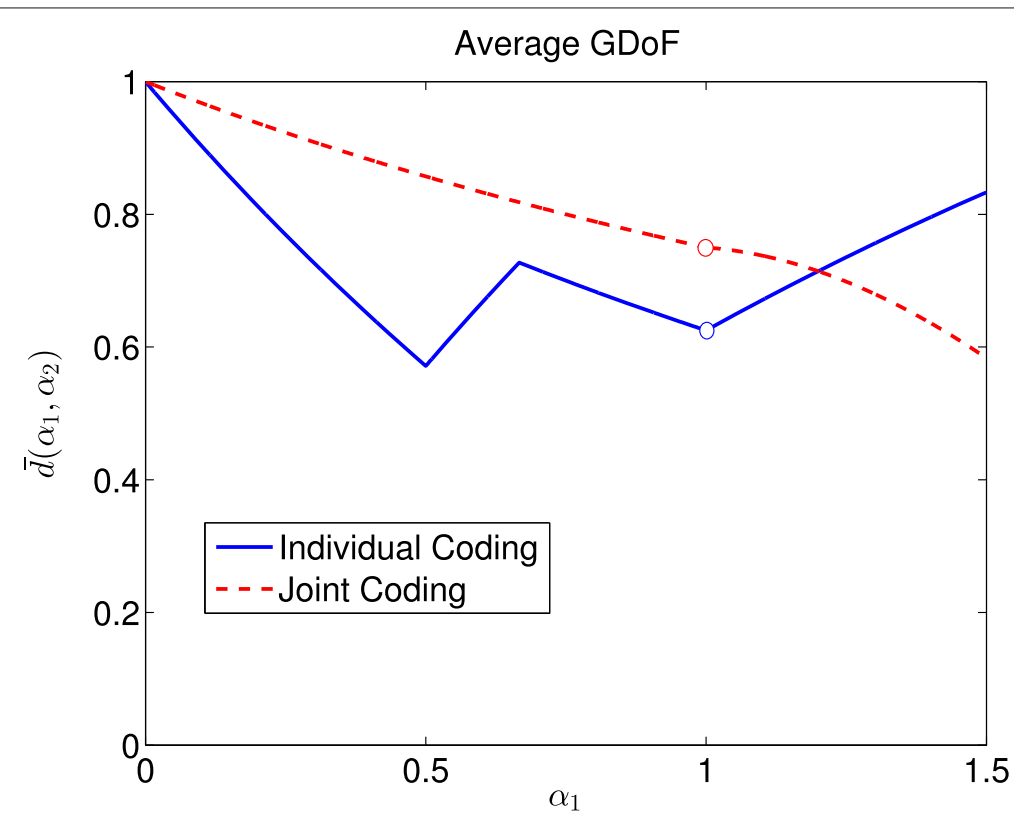

Fig. 9 Average achievable GDoFs with different $\alpha_{1}$ and fixed $\alpha_{2}$, where $\alpha_{2}=3$ 


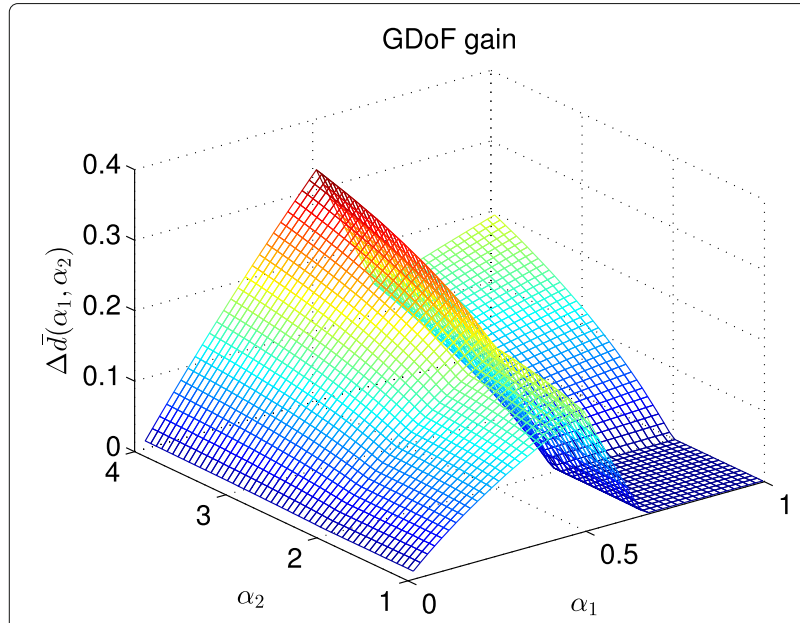

Fig. 10 GDoF gains with different $\alpha_{1}$ and $\alpha_{2}$

interference subchannels were effectively used to assist interference mitigation in weak interference subchannels. We proved that this joint coding scheme can achieve the sum capacity in two-user case and can achieve the GDoF in multi-user case. Numerical results demonstrated substantial GDoF gains over the individual coding scheme.

\section{Acknowledgements}

This work was supported by the National Natural Science Foundation of China under Grants 61371077 and 61429101. The authors would like to thank the anonymous reviewers for their constructive comments, which helped a lot to improve the presentation of this paper.

\section{Competing interests}

The authors declare that they have no competing interests.

\section{Publisher's Note}

Springer Nature remains neutral with regard to jurisdictional claims in published maps and institutional affiliations.

Received: 9 November 2015 Accepted: 6 March 2017

Published online: 16 March 2017

\section{References}

1. ST Chung, JM Cioffi, The capacity region of frequency-selective Gaussian interference channels under strong interference. IEEE Trans. Commun. 55(9), 1812-1821 (2007)

2. W Yu, G Ginis, JM Cioffi, Distributed multiuser power control for digital subscriber lines. IEEE J. Sel. Areas Commun. 20(5), 1105-1115 (2002)

3. W Yu, R Lui, Dual methods for nonconvex spectrum optimization of multicarrier systems. IEEE Trans. Commun. 54(7), 1310-1322 (2006)

4. VR Cadambe, SA Jafar, Parallel Gaussian interference channels are not always separable. IEEE Trans. Inf. Theory. 55(9), 3983-3990 (2009)

5. X Shang, B Chen, G Kramer, HV Poor, Noisy-interference sum-rate capacity of parallel Gaussian interference channels. IEEE Trans. Inf. Theory. 57(1), 210-226 (2011)

6. H Sun, SA Jafar, in ISIT. On the optimality of treating interference as noise for parallel deterministic interference networks, (2014)

7. L Sankar, X Shang, E Erkip, HV Poor, Ergodic fading interference channels: sum-capacity and separability. IEEE Trans. Inf. Theory. 57(5), 2605-2626 (2011)

8. TS Han, K Kobayashi, A new achievable rate region for the interference channel. IEEE Trans. Inf. Theory. 27(1), 49-60 (1981)

9. RH Etkin, DNC Tse, H Wang, Gaussian interference channel capacity to within one bit. IEEE Trans. Inf. Theory. 54(12), 5534-5562 (2008)
10. SA Jafar, S Vishwanath, Generalized degrees of freedom of the symmetric Gaussian $K$ user interference channel. IEEE Trans. Inf. Theory. 56(7), 3297-3303 (2010)

11. SA Jafar, Topological interference management through index coding. IEEE Trans. Inf. Theory. 60(1), 529-568 (2014)

12. C Suh, DNC Tse, Feedback capacity of the Gaussian interference channel to within 2 bits. IEEE Trans. Inf. Theory. 57(5), 2667-2685 (2011)

13. S Mohajer, R Tandon, HV Poor, On the feedback capacity of the fully connected $K$-user interference channel. IEEE Trans. Inf. Theory. 59(5), 2863-2881 (2013)

14. Y Wang, Y Tian, C Yang, C Sun, in PIMRC. Multi-carrier cooperated interference cancelation in heterogeneous cellular networks, (2013)

15. N Hou, Y Tian, C Yang, in ICCC. Multi-carrier cooperated interference cancellation via macro-BS broadcasting in HetNet, (2014)

16. P Mukherjee, R Tandon, S Ulukus, in Allerton Conf. Even symmetric parallel linear deterministic interference channels are inseparable, (2013)

17. S Mishra, I Wang, S Diggavi, in ISIT. Harnessing bursty interference in multicarrier systems with feedback, (2014)

18. AA El Gamal, MHM Costa, The capacity region of a class of deterministic interference channels. IEEE Trans. Inf. Theory. 28(2), 343-346 (1982)

19. S Saha, R Berry, in Allerton Conf. Sum-capacity of a class of $K$-user Gaussian interference channels within $O(K)$ bits, (2011)

20. D Maamari, D Tuninetti, N Devroye, Multi-user cognitive interference channels: a survey and new capacity results. IEEE Trans. Cogn. Commun. Netw. 1(1), 29-44 (2015)

21. S Gherekhloo, A Chaaban, A Sezgin, Cooperation for interference management: a GDoF perspective. IEEE Trans. Inf. Theory. 62(12), 6986-7029 (2016)

22. R Kolte, A Özgür, H Permuter, Multicoding schemes for interference channels. IEEE Trans. Inf. Theory. 62(9), 4936-4952 (2016)

23. M Kiamari, A Avestimehr, in Allerton Conf. Are generalized cut-set bounds tight for the deterministic interference channel? (2015)

24. S Avestimehr, S Diggavi, DNC Tse, Wireless network information flow: a deterministic approach. IEEE Trans. Inf. Theory. 57(4), 1872-1905 (2011)

25. G Bresler, A Parekh, DNC Tse, The approximate capacity of the many-to-one and one-to-many Gaussian interference channels. IEEE Trans. Inf. Theory. 56(9), 4566-4592 (2010)

\section{Submit your manuscript to a SpringerOpen ${ }^{\circ}$ journal and benefit from:}

- Convenient online submission

- Rigorous peer review

- Immediate publication on acceptance

- Open access: articles freely available online

- High visibility within the field

- Retaining the copyright to your article

Submit your next manuscript at springeropen.com 